\title{
A HARNACK INEQUALITY FOR NONLINEAR EQUATIONS
}

\author{
BY JAMES SERRIN
}

Communicated by Lawrence Markus, March 18, 1963

Recently Moser has obtained a Harnack inequality for linear divergence structure equations with $n>2$ variables. In this note we indicate how a similar procedure can be used also for nonlinear equations; in fact the equations in question need not even satisfy the usual ellipticity conditions. As applications of our main result, we obtain, among other things, an a priori estimate for the Hölder continuity of solutions and the general asymptotic behavior of positive solutions at an infinite singularity.

Consider specifically equations of the form

$$
\operatorname{div} A\left(x, u, u_{x}\right)=B\left(x, u, u_{x}\right), \quad x=\left(x_{1}, \cdots, x_{n}\right) \in D,
$$

where $D$ is a bounded open set in Euclidean $n$-space. In this equation $A$ is a given vector function of $x, u, u_{x}, B$ is a given scalar function of the same variables, and

$$
\operatorname{div} A=\sum_{1}^{n} \frac{\partial A_{i}}{\partial x_{i}}, \quad u_{x}=\left(\frac{\partial u}{\partial x_{1}}, \ldots, \frac{\partial u}{\partial x_{n}}\right) .
$$

The structure of equation (1) is determined by the functions $A(x, u, p)$ and $B(x, u, p)$. We assume that they are measurable in $x$ and continuous in $u$ and $p$, and that they satisfy inequalities of the form

$$
\begin{aligned}
& |A| \leqq a|p|^{\alpha-1}+b|u|^{\alpha-1}+e, \\
& |B| \leqq c|p|^{\alpha-1}+d|u|^{\alpha-1}+f, \\
& p \cdot A \geqq a^{-1}|p|^{\alpha}-d|u|^{\alpha}-g,
\end{aligned}
$$

for $x \in D$ and all values of $u$ and $p$. Here $\alpha, 1<\alpha<n$, is a fixed exponent, $a$ is a positive constant, and $b$ through $g$ are measurable functions on $D$ in the respective Lebesgue classes

$$
b, e \in L_{n /(\alpha-1)} ; \quad c \in L_{n /(1-\epsilon)} ; \quad d, f, g \in L_{n /(\alpha-\epsilon)},
$$

$\epsilon$ being some positive number less than or equal to one. [We can also treat the case $\alpha=n$. The case $\alpha>n$, moreover, is somewhat easier and can be handled by means of Morrey's lemma. For simplicity and brevity of presentation we shall here restrict consideration to the range $1<\alpha<n$, as indicated above.]

The generality of these assumptions requires that equation (1) be interpreted in a weak sense. Let $u=u(x)$ be a function having strong 
derivatives which are locally of class $L_{\alpha}$ over $D$, that is, $u \in H_{\alpha}^{1}(D)$. Then $u$ will be called a weak solution of (1) in $D$ if

$$
\int\left(\phi_{x} \cdot A+\phi B\right) d x=0
$$

for any continuously differentiable function $\phi=\phi(x)$ with compact support in $D$. Obviously any function which satisfies (1) in the classical sense would also be a solution in the sense just defined, though of course not conversely.

Before proceeding further it is worth indicating several classes of equations which fall into the general category above. Suppose first that

$$
\begin{aligned}
A_{i} & =a_{i j} p_{j}+b_{i} u+e_{i}, \\
B & =c_{j} p_{j}+d u+f,
\end{aligned}
$$$$
\text { ( } j \text { summed), }
$$

where $a_{i j}=a_{i j}(x)$ obeys the ellipticity condition

$$
\lambda^{-1} \xi^{2} \leqq a_{i j} \xi_{i} \xi_{j} \leqq \lambda \xi^{2}, \quad \lambda=\text { const }>0,
$$

and the coefficients $b_{i}$ through $f$ fall into the Lebesgue classes

$$
b_{i}, c_{i}, e_{i} \in L_{n /(1-\epsilon)} ; \quad d, f \in L_{n /(2-e) .}
$$

One checks easily that the various conditions (2) are met with $\alpha=2$, for example

$$
p \cdot A=a_{i j} p_{i} p_{j}+b_{i} p_{i} u+e_{i} p_{i} \geqq(2 \lambda)^{-1}|p|^{2}-\lambda|b|^{2}|u|^{2}-\lambda|e|^{2}
$$

and both $|b|^{2}$ and $|e|^{2}$ are in $L_{n /(2-\epsilon)}$ as required by (3). This case essentially coincides with the class of linear equations studied by Morrey in reference [6]; moreover, when $b_{i}$ through $f$ are identically zero we have precisely the situation considered by Moser in [8]. As a second example, consider the variational problem

$$
\int_{D} F\left(x, u, u_{x}\right) d x=\text { Minimum, }
$$

where the integrand $F(x, u, p)$ is convex in $p$ and subject to the conditions

$$
\begin{aligned}
F & \geqq a^{-1}|p|^{\alpha}-d|u|^{\alpha}-g, \\
\left|F_{p}\right| & \leqq a|p|^{\alpha-1}+b|u|^{\alpha-1}+e, \\
\left|F_{u}\right| & \leqq c|p|^{\alpha-1}+d|u|^{\alpha-1}+f .
\end{aligned}
$$

The coefficients in (6) are assumed to satisfy (3), and in addition we suppose that $F(x, 0,0) \in L_{n /(\alpha-\epsilon)}$. The Euler equation for (5) has the 
form (1) with $A=F_{p}, B=F_{u}$. One checks that the various inequalities (2) are satisfied. Specifically,

$$
F(x, u, 0)=F(x, 0,0)+\int_{0}^{u} F_{u}(x, u, 0) d u \leqq \bar{d}|u|^{\alpha}+\bar{g}
$$

so that, using the convexity of $F$,

$p \cdot F_{p} \geqq F(x, u, p)-F(x, u, 0) \geqq a^{-1}|p|^{\alpha}-(d+\bar{d})|u|^{\alpha}-(g+\bar{g})$.

It should be observed that conditions (6) are similar to those assumed in the papers $[4 ; 7]$, etc.

As a final example, we note that certain equations which nominally do not fall into the category above, can in fact be considered as special cases. In particular, suppose one replaces (2) by

$$
\begin{aligned}
& |A| \leqq a\left(|p|^{\alpha-1}+|u|^{(\alpha-1) / \mu}+e\right), \\
& |B| \leqq a\left(|p|^{\alpha-\mu}+|u|^{(\alpha-\mu) / \mu}+f\right), \\
& p \cdot A \geqq a^{-1}|p|^{\alpha}-a\left(|u|^{\alpha / \mu}+g\right),
\end{aligned}
$$

where $\mu=(n-\alpha) / n+\epsilon$. For any given solution of (1) for this case, we may set

$$
b(x)=a|u|^{(\alpha-1)(1-\mu) / \mu}, \quad c(x)=a\left|u_{x}\right|^{1-\mu}, \quad d(x)=a|u|^{\alpha(1-\mu) / \mu},
$$

and it is easily verified (since $u_{x} \in L_{\alpha}, u \in L_{\alpha_{n} /(n-\alpha)}$ ) that conditions (3) are in fact satisfied. Thus the conclusions of the following Theorems 1 through 3 remain valid, with the exceptions that the coeffcients $k, K$, etc. now depend also on the $W_{\alpha}^{1}$ norm of the solution.

Now let $Q(P, R)$ denote the cube of half-edge $R$ and center $P$ in Euclidean $x$-space. The main result of the paper is the following

TheOREM 1. Let $u$ be a (weak) solution of (1) in D. Suppose that $u$ is positive in some cube $Q(P, 4 R)$ contained in $D$. Then in $Q(P, R)$ we have

$$
\max u \leqq k\left(\min u+k^{\prime}\right){ }^{1}
$$

where $k$ and $k^{\prime}$ are constants depending only on the structure of equation (1). In particular

$$
k=k\left(\alpha, n, \epsilon ; a,\|b\|, R^{\epsilon}\|c\|, R^{\star}\|d\|\right)
$$

and

$$
k^{\prime}=\left(\|e\|+R^{\epsilon}\|f\|\right)^{1 /(\alpha-1)}+\left(R^{\ell}\|g\|\right)^{1 / \alpha},
$$

${ }^{1}$ Here max and min are to be interpreted as the essential maximum and the essential minimum. 
the norms of the coefficients $b$ through $g$ being taken in the respective Lebesgue spaces (3).

Theorem 1 is proved essentially by the method of reference [8], with strong use being made of the general Sobolev inequalities and the lemma of John and Nirenberg. The first step of the proof involves the substitutions $\bar{u}=u+k^{\prime}, \bar{x}=x / R$, which reduces the theorem to the case when $R=1$ and $e=f=g=0$. We then introduce the test function $\phi=\eta^{\alpha} u^{\beta}$ into (4) and proceed rather as in Moser's proof. [It should be observed that before this procedure can be applied it is necessary to know that $u$ is bounded. However, a preliminary argument may be used to establish the following result: Let $u$ be a weak solution of (1) in $D$. Then $u$ is essentially bounded on any compact subset $D^{\prime}$ of $D$, the bound depending only on the structure of equation (1), the sets $D$ and $D^{\prime}$, and the $L_{\alpha}$ norm of $u$ over $\left.D .^{2}\right]$ Complete details of the method will appear in a subsequent paper. The following result is an obvious consequence of Theorem 1.

Theorem 2. If $u$ is a positive solution of (1) in $D$, and if $D^{\prime}$ is any compact set in $D$, then

$$
\max _{D^{\prime}} u \leqq K\left(\min _{D^{\prime}} u+K^{\prime}\right)
$$

where $K$ and $K^{\prime}$ depend only on the structure of equation (1) and the domains $D, D^{\prime}$.

Suppose now that $e$ is in a more restrictive Lebesgue class than originally assumed, namely $e \in L_{n /(\alpha-1-\epsilon)}(0<\epsilon \leqq \alpha-1)$. Then clearly $k^{\prime} \leqq$ const $R^{\epsilon / \alpha}$ in (7), where the constant depends only on the norms of $e, f$, and $g$ and on the diameter of $D$. This being the case, it is easy to show that $u$ must be Hölder continuous, thus:

Theorem 3. Let $u$ be a solution of (1) in $D$ and let $D^{\prime}$ be a compact set in $D$. Then after suitable redefinition of $u$ on a set of measure zero, $u$ is Hölder continuous on $D^{\prime}$ with coefficient and exponent depending only on the structure of equation (1), on the domains $D$ and $D^{\prime}$, and on the $L_{\alpha}$ norm of $u$ over $D$.

A more refined application of Theorem 1 is found in the study of solutions having isolated singular points. For simplicity in the discussion we shall consider only a special case of equation (1), namely

2 This may be assumed finite. Otherwise we can replace $D$ by a suitable subdomain $D^{*}$ with compact closure in $D$. 


$$
\operatorname{div} A\left(x, u_{x}\right)=0,
$$

where $|A| \leqq a|p|^{\alpha-1}+e, p \cdot A \geqq a^{-1}|p|^{\alpha}-g$, and $e$ and $g$ are respectively in $L_{n /(\alpha-1-\epsilon)}$ and $L_{n /(\alpha-\epsilon)}$. Moreover, we shall assume that equation (8) has the following additional properties:

1. For all $x \in D$ and all values of $p$ and $q$,

$$
(p-q) \cdot(A(x, p)-A(x, q)) \geqq 0,
$$

with equality holding if and only if $p=q$.

2. For smooth boundaries and smooth boundary data there exist continuously differentiable (weak) solutions of (8) taking on the given boundary values.

3. Let $\Gamma$ denote a spherical annulus in $D$. By assumption 2 there exists a solution $v$ of $(8)$ taking the boundary value zero on the outer circumference and $m \geqq 0$ on the inner circumference. Also, by virtue of (9) a weak maximum principle holds for the difference of any two solutions. Consequently, at any point $P$ in $\Gamma$ the values $v(P)$ increase monotonically with $m$. As the final assumption, we suppose that $v(P)$ tends to infinity as $m$ does.

The structure noted above is obviously available in the case of linear equations. A more sophisticated situation where the assumptions can be verified occurs when $A=A(p)$ is of class $C^{2}$,

$$
|A| \leqq a\left(|p|^{\alpha-1}+1\right), \quad p \cdot A \geqq a^{-1}|p|^{\alpha}-a,
$$

and the derivatives $A_{i, k}$ of $A$ satisfy $\lambda_{1}(p) \xi^{2} \leqq A_{i, k}(p) \xi_{i} \xi_{k} \leqq \lambda_{2}(p) \xi^{2}$, where $\lambda_{1}$ and $\lambda_{2}$ are positive continuous functions and $\lambda_{2} / \lambda_{1} \leqq$ const. By applying the mean value theorem, it is easy to verify the relation (9). The existence theorem needed to justify the second assumption has been given by Gilbarg in two recent papers, [1] and [2]. The final condition can be proved by standard comparison arguments.

THEOREM 4. Let $u$ be a continuous non-negative solution of (8) in the set $D-\{0\}$, and suppose that the preceding assumptions 1 through 3 are satisfied. Then either $u$ is uniformly Hölder continuous in the neighborhood of 0 , or else there exist positive constants $C$ and $C^{\prime}$ such that the inequality

$$
C r^{\kappa} \leqq u \leqq C^{\prime} r^{k}, \quad \kappa=\frac{\alpha-n}{\alpha-1},
$$

holds in the neighborhood of 0.

That is, a solution with an isolated singularity either satisfies the maximum principle or (if it is bounded on one side) has precisely the order of growth $r^{(\alpha-n) /(\alpha-1)}$ at the singularity. When (8) is linear, we 
have $\alpha=2$ and the singularity is of order $r^{2-n}$ as is well known $[5 ; 9]$. A somewhat weaker version of Theorem 4 was given earlier by the writer [10]. For linear equations of the form $a_{i j} \partial^{2} u / \partial x_{i} \partial x_{j}=0$ there are corresponding isolated singularity theorems, requiring however some continuity of the coefficients, and not necessarily providing an explicit order of growth at the singularity $[1 ; 3]$.

The proof of Theorem 4 is fairly detailed, and cannot be given here. We require in particular the preceding Harnack inequalities, a generalization of the capacitary potential techniques of [5] and the isolated singularity theorem of reference [10]. In addition, an important step in the argument uses the fact that the function $h=m r^{k}+m^{\prime}$, $m, m^{\prime}=$ constants, is a solution of the variational problem (5) for $F=|p|^{\alpha}$.

In conclusion, one may ask whether there exist any solutions of (8) having the asymptotic behavior (10) at an isolated singularity. Under the present general conditions we have been unable to establish this, but if somewhat stronger regularity conditions are placed on the function $A(x, p)$ then the existence of such solutions can be obtained.

\section{REFERENCES}

1. D. Gilbarg, Some local properties of elliptic equations, Proc. Sympos. Pure Math., Vol. 4, Partial Differential Equations, pp. 127-142, Amer. Math. Soc., Providence, R. I., 1961.

2. - Boundary value problems for non-linear elliptic equations, Nonlinear Problems (edited by R. E. Langer), pp. 151-160, Univ. of Wisconsin Press, Madison, Wis., 1963.

3. D. Gilbarg and J. Serrin, On isolated singularities of solutions of second order elliptic differential equations, J. Analyse Math. 4 (1955-56), 309-340.

4. O. A. Ladyzhenskaya and N. Uraltseva, Quasi-linear elliptic equations and variational problems with many independent variables, Uspehi Mat. Nauk 16 (1961), 19-92; translated in Russian Math. Surveys 16 (1961), 17-91.

5. W. Littman, G. Stampacchia and H. Weinberger, Regular points for elliptic equations with discontinuous coefficients (to appear).

6. C. B. Morrey, Jr., Second order elliptic equations and Hölder continuity, Math. Z. 72 (1959), 146-164.

7. - Existence and differentiability theorems for variational problems for multiple integrals, Partial Differential Equations and Continuum Mechanics, pp. 241-270, Univ. of Wisconsin Press, Madison, Wis., 1961.

8. J. Moser, On Harnack's theorem for elliptic differential equations, Comm. Pure Appl. Math. 14 (1961), 577-591.

9. H. Royden, The growth of a fundamental solution of an elliptic divergence structure equation, Studies in Mathematical Analysis and Related Topics: Essays in honor of G. P6lya, pp. 333-340, Stanford Univ. Press, Stanford, Calif., 1962.

10. J. Serrin, Dirichlet's principle in the calculus of variations, Proc. Sympos. Pure Math., Vol. 4, Partial Differential Equations, pp. 17-22, Amer. Math. Soc., Providence, R. I., 1961.

University OF MinNesota 